\title{
Editorial
}

\section{The Challenges of Screening for Substance Use in Pregnant Women: Commentary on the 4P'S Plus Tool}

\section{Hendree E. Jones, PhD}

Journal of Perinatology (2005) 25, 365-367. doi:10.1038/sj.jp.7211293

Chasnoff and colleagues tested the ability of a five-item brief screening instrument, $4 \mathrm{P}^{\prime}$ ' Plus ${ }^{\odot}$ to detect substance use in pregnant women receiving prenatal care. The patient population was diverse in both geographical location and socioeconomic status. Results showed that $21.9 \%$ of patients admitted tobacco use and 20\% reported alcohol use in the month prior to pregnancy awareness. It was interesting to note that approximately $50 \%$ of the women who used alcohol in the month before pregnancy awareness spontaneously quit. Of women continuing to consume alcohol, the majority drank less than 2 days a week. The rate of use of cocaine, heroin and methamphetamines was $1 \%$ after pregnancy awareness. In contrast to the spontaneous quit rates observed with alcohol and illicit drug use, almost all women continued smoking through the rest of pregnancy. The strengths of this study that merit comment are numerous; however, three are discussed. Three areas of critique are also presented.

First, this study provides a very sensitive screen for prenatal substance use. The screen is a major step forward in acknowledging the stigma associated with admitting prenatal substance use. Asking about both parental and partner use of alcohol and drugs, risk factors of substance abuse for women, is less stigmatizing for women to endorse than their own substance use. Seemingly, women would be more likely to admit someone else's substance use than their own. Focusing on prepregnancy use may also increase the likelihood a patient will accurately endorse substance use. Asking about prepregnancy use of tobacco and alcohol in terms of numbers of cigarettes and alcohol drinks consumed is a clinically sensitive way to "normalize" substance use without stigmatizing patients. It is also likely to yield more accurate answers than asking if the patient uses alcohol or tobacco, with a positive response followed by questions quantifying use.

Second, the authors are to be applauded for using the sensitive combination of screening followed by assessment. Their data are similar to those of Horrigan and Piazza (1999), ${ }^{1}$ who reported that

Department of Psychiatry and Behavioral Sciences, Johns Hopkins University School of Medicine, Baltimore, MD 21224, USA.

Address correspondence and reprint requests to Hendrée E. Jones, PhD, Johns Hopkins Bayview Medical Campus, D-3-East, 4940 Eastern Ave, Baltimore, MD 2122, USA. a substance abuse screen followed by targeted diagnostic assessment was 100\% accurate in detecting alcohol and cocaine use and more sensitive than using one self-report measure with urine toxicology screening. Chasnoff and colleagues' data are yet another important example highlighting the limitations of relying solely on biological testing (e.g., urine toxicology). Self-report measures have the added advantage over biological specimen testing in that by disclosing information or remaining silent, women are providing or withholding consent to participate in medical care related to substance use. However, there are important limitations of self-report that cannot be overlooked. Self-report can be unreliable because of poor recall, fear of stigma and fear of criminal prosecution or involvement of child welfare services. Thus, efforts are needed to create environments that encourage, not impede, accurate self-report.

Third, the results of this study are provocative. Chasnoff and colleagues showed that those in the wealthiest community had the highest rate of reporting any substance use (50\%) during pregnancy. These data are striking and highlight the importance of screening for substance use in all prenatal patient populations, not just the poor and uninsured patients. Patients of all economic levels can benefit by having the health-care practitioner provide the opportunity for women to ask questions about substance use. Tailoring public health messages into specific and personal messages are also more likely to evoke behavioral change. ${ }^{2}$ In reading the study, one cannot help but wonder how much more prenatal substance use could be prevented if screening and assessment efforts focused on nonpregnant women of child-bearing age before they become pregnant and after they deliver. Perhaps substance use screening should be expanded to the gynecological check-up and be as routine as yearly cervical cancer screening. The need for continued substance use screening postpartum is clear as many women return to harmful substance using patterns shortly after delivery. ${ }^{3,4}$ However, one also wonders about the contextual issues in which these messages for behavior change are given. Often, health-care workers are reluctant to ask about substance use because they lack the knowledge and resources to respond to a positive answer or want to avoid the understandable frustration when patients refuse to change harmful behaviors. Further, a woman can spend hours at the clinic waiting to be seen, yet her visit with the health-care practitioner is limited to a quick examination and perhaps a lecture about what she is doing wrong (smoking, drinking, too much or little exercise or weight gain). ${ }^{5}$ Although repeatedly confronting women with the poor health 
choices they are making and potentially imposing punitive consequences for not remaining abstinent from substances may appear to be attractive methods for producing behavior change, these actions often result in pushing patients away from needed medical treatment. Thus, how messages about behavior change are given is as important as the content of the message. The high rate of substance use reported by the economically advantaged patients also calls into question the importance of understanding the context in which prenatal drug exposure alters development. It is well established that wide variations exist in developmental outcomes of substance-exposed children. The factors determining child outcome are complex and omni-faceted. This study provides an important population of children and families to follow to help tease apart the contributions of prenatal substance exposure and socioeconomic status. It is known that poverty alone and in combination with substance exposure has negative consequences on child intelligence and behavior, ${ }^{6}$ yet few studies have had the potential opportunity to closely follow such a resource-rich patient sample as that of Chasnoff and colleagues.

The strengths of the sensitive $4 P^{\prime}$ s Plus ${ }^{\mathbb{C}}$ screening instrument may also be viewed as weaknesses if one ultimate goal is to reduce the burden of the health practitioner in quickly and accurately identifying, treating or referring the patient with problem substance use to appropriate care.

First, referring a patient for more intensive and time-consuming diagnostic assessment (time nor details of this assessment were provided in the article) if she reports ingesting one standard drink (12 oz beer or $5 \mathrm{oz}$ glass of wine) before pregnancy awareness may be an over use of resources. What if this woman reported having one glass of champagne on a special occasion before pregnancy awareness? Is this woman "at risk"? Should she and the healthcare provider be asked to complete the full assessment if negative effects on children are seen at much higher ingestion rates of two to three drinks a day near the time of conception ${ }^{7}$ or averaging one drink per week during pregnancy? ? $^{8}$ Although it is clearly acknowledged that there is no level of alcohol consumption deemed "safe" during pregnancy and abstinence is the best behavior, care needs to be taken in balancing between overlooking and overfocusing on light drinking. While education about health behaviors during pregnancy can help improve a patient's behavioral choices, focusing too much on a rare behavior may be a disincentive for women to attend prenatal care. It may also be a disincentive for health-care providers to screen for substance abuse if they will have to administer a lengthy assessment to a woman reporting consumption of one alcoholic drink.

Second, reporting the quantity and frequency of substance use would have strengthened the paper. By reporting only percentages of pregnant women endorsing questions and categorizing days of alcohol use, it is not clear if a "continued drinker" is a woman who drank one time (say one drink) or multiple times post pregnancy awareness. Alternatively, that one-day drinker could have drunk to the point of severe intoxication and/or passing out. Thus, it seems that reporting drinking levels in ounces of absolute alcohol per day or per week would provide a more sensitive indication as to the level of risk of harm to the fetus than days of use. This seems especially important as animal models have shown that peak blood alcohol level rather than total amount of alcohol consumed may represent the "critical dose" or threshold of alcohol above which an adverse effect may be seen. ${ }^{9}$

Third, the complete reliance on self-report data only identifies patients who want to be revealed. Given that many studies of pregnant patients show higher rates on biomarkers of drug-positive toxicologies than self-reported drug use, ${ }^{10-13}$ it would have been interesting to see results of toxicology screening on the entire sample and examine the agreement between toxicology, screening and assessment results. Although alcohol use is notoriously difficult to detect with urine toxicologies, and appears to be less accurate in the presence of pregnancy hormones, ${ }^{14}$ it would have been interesting to see toxicology testing from neonatal samples like meconium, which is able to detect substance use during a long window of time in pregnancy.

In summary, the $4 P^{\prime}$ S Plus ${ }^{(\mathcal{C}}$ is an important step in secondary prevention focused on identifying and decreasing substance use during pregnancy. The authors are to be congratulated on creating this important tool. This prevention effort will only be effective if patients self-disclose substance use. To maximize the likelihood of self-reporting of stigmatizing behavior, health-care settings must be seen as supportive and nonpunitive environments. Health-care practitioners need the knowledge to detect and refer patients to needed substance treatment services. Finally, it is unfortunate that resources for such an important health care problem are limited. One can only imagine the hundreds of thousands of mothers and children whose lives would be improved if the proportion of federal funds spent on drug supply reduction/interdiction efforts $(67 \%$, 19.2 billion) and prevention and treatment (33\%) were reversed.

\section{References}

1. Horrigan TJ, Piazza N. The substance abuse subtle screening inventory minimizes the need for toxicology screening of prenatal patients. J Subst Abuse Treat 1999;17:243-7.

2. Minor MJ, Van Dort B. Prevention research on the teratogenic effects of alcohol. Prev Med 1982;11:346-59.

3. Substance Abuse and Mental Health Services Administration. National Household Survey on Drug Abuse (NHSDA) report. Tobacco and Alcohol Use Among Pregnant Women Summary of findings from the 1999 National Household Survey on Drug Abuse. NHSDA Series: H-12, DHHS Publication No. SMA 00-3466. Rockville, MD; 2001.

4. Substance Abuse and Mental Health Services Administration. National Household Survey on Drug Abuse (NHSDA) report. Pregnancy and Illicit Drug Use Summary of findings from the 1999 National Household Survey on Drug Abuse. NHSDA Series: H-12, DHHS Publication No. SMA 00-3466. Rockville, MD; 2001. 
5. Lazarus ES. Falling through the cracks: contradictions and barriers to care in a prenatal clinic. Med Anthropol 1990;12:269-87.

6. Lester BM, Andreozzi L, Appiah L. Substance use during pregnancy: time for policy to catch up with research. Harm Reduct J 2004;1:5.

7. Sokol RJ, Martier SS, Ager JW. The T-ACE questions: practical prenatal detection of risk-drinking. Am J Obstet Gynecol 1989;160:863-8.

8. Sood B, Delaney-Black V, Covington C, et al. Prenatal alcohol exposure and childhood behavior at age 6 to 7 years: I. dose-response effect. Pediatrics 2001;108:E34.

9. West JR, Goodlett CR, Brandt JP. New approaches to research on the longterm consequences of prenatal exposure to alcohol. Alcohol Clin Exp Res 1990;14:684-9.
10. Frank DA, Zuckerman BS, Amaro H, et al. Cocaine use during pregnancy: prevalence and correlates. Pediatrics 1988;82:888-95.

11. Schutzman DL, Frankenfield-Chernicoff M, Clatterbaugh HE, Singer J Incidence of intrauterine cocaine exposure in a suburban setting. Pediatrics 1991;88:825-7.

12. Ostrea Jr EM, Brady M, Gause S, Raymundo AL, Stevens M. Drug screening of newborns by meconium analysis: a large-scale, prospective, epidemiologic study. Pediatrics 1992;89:107-13.

13. Lester BM, ElSohly M, Wright LL, et al. The Maternal Lifestyle Study: drug use by meconium toxicology and maternal self-report. Pediatrics 2001;107:309-17.

14. Coles C. Critical periods for prenatal alcohol exposure: evidence from animal and human studies. Alcohol World Health Res. NIAAA 1994;18:22-9. 